\title{
Hallazgos radiológicos del compromiso torácico de las enfermedades dermatológicas: lo que los dermatólogos debemos saber
}

\author{
Felipe Aluja-Jaramillo후 Jairo Mahecha²; Cesar González-Ardila
}

\section{RESUMEN}

Introducción: existe una gran relación entre las enfermedades dermatológicas y sus manifestaciones torácicas. Su detección temprana no solo es importante para orientar el diagnóstico con su consecuente correlación con las manifestaciones cutáneas, también ayuda a dirigir el manejo clínico apropiado, principalmente el de la enfermedad pulmonar intersticial asociada a las patologías del tejido conectivo. El objetivo de esta revisión es mostrar la relación clínica y radiológica de las principales entidades infecciosas, inflamatorias, del tejido conectivo, hereditarias y adquiridas de tipo dermatológico que presentan compromiso pulmonar asociado. Discusión: es importante la realización de una tomografía computarizada multidetectora en la valoración de la patología del parénquima pulmonar, que puede pasar desapercibida en la radiografía de tórax. El dermatólogo debe conocer la terminología radiológica y los hallazgos radiológicos básicos.

PALABRAS CLAVE: Enfermedades de la piel; Enfermedades del tejido conjuntivo; Enfermedades pulmonares intersticiales; Tejido conectivo; Tomografía computarizada multidetectora.

1. Departamento de Radiología, Hospital Universitario San Ignacio-Pontificia Universidad Javeriana, Country Scan LTDA, Bogotá, Colombia. ORCID https://orcid.org/oooo-0002-3093-2509

2. Pontificia Universidad Javeriana, Bogotá, Colombia. ORCID https://orcid.org/oooo-0002-0347-3263

3. Médico Dermatólogo, Bogotá, Colombia. ORCID https://orcid.org/oooo-0002-5608-9054

Correspondencia: Felipe Aluja-Jaramillo; email: faluja@javeriana.edu.co

Recibido: 22/08/20; aceptado: 25/11/20

Cómo citar: Aluja, F; Mahecha, J; González, C. Hallazgos radiológicos del compromiso torácico de las enfermedades dermatológicas: lo que los dermatólogos debemos saber. Rev Asoc Colomb Dermatol. Vol 28(4): octubre - diciembre, 2020, 325-339.

DOI: https://doi.org/10.29176/2590843X.1552

Financiación: ninguna, conflictos de interés: ninguno 


\section{RADIOLOGICAL FINDINGS OF THORACIC INVOLVEMENT IN DERMATOLOGIC DISEASES: WHAT DERMATOLOGISTS SHOULD KNOW}

\section{SUMMARY}

Introduction: there is a great relationship between dermatological diseases and their thoracic manifestations. Its early detection is important, not only to guide the diagnosis with its consequent correlation with skin manifestations, but also to help direct the most appropriate clinical management, such as interstitial lung disease associated with connective tissue pathologies. Within this article there is a review in relation to the clinical and radiological correlation of the main entities both infectious, inflammatory, connective tissue diseases, hereditary and acquired diseases. Discussion: It's important performing a multidetector computed tomography in the detection of pulmonary parenchymal pathology that may go unnoticed in the chest radiograph. The dermatologist should know the radiological terminology and basic radiological findings.

KEY WORDS: Connective tissue diseases; Connective tissue diseases; Interstitial lung diseases; Multidetector computed tomography; Skin diseases.

\section{INTRODUCCIÓN}

Existen algunas patologías que se caracterizan por la afectación simultánea de la piel y el pulmón con diferentes implicaciones clínicas según su compromiso. Etiologías infecciosas, como la endocarditis bacteriana o el síndrome sinovitis, acné, pustulosis, hiperostosis y osteomielitis (SAPHO); inflamatorias, como la sarcoidosis; enfermedades del tejido conectivo, como la polimiositis y la dermatomiositis, la esclerosis sistémica, el síndrome de Sjögren, el lupus eritematoso sistémico, la artritis reumatoide y la psoriasis; entidades hereditarias, como la neurofibromatosis tipo 1 y la esclerosis tuberosa; o adquiridas, como el sarcoma de Kaposi, todas pueden presentar compromiso en ambos órganos durante el curso de la enfermedad.

Nuestro objetivo es introducir la correlación de las manifestaciones cutáneas y las manifestaciones radiológicas torácicas, especialmente en tomografía de tórax de alta resolución (TACAR) o en la tomografía computarizada multidetectora con contraste de tórax (TCMD), centrándonos en las manifestaciones más frecuentes que pueden aportar información valiosa para el dermatólogo al momento del diagnóstico y seguimiento de estas patologías.
PATRONES RADIOLÓGICOS ASOCIADOS A LAS ENFERMEDADES DERMATOLÓGICAS

Para comprender el compromiso torácico debemos iniciar por conocer los términos radiológicos básicos que permitan su compresión. Una de las formas de compromiso habitualmente descrito en el tórax es la enfermedad pulmonar intersticial (EPI), la cual comprende un grupo diverso de alteraciones en el parénquima pulmonar y que comparten presentaciones radiológicas, patológicas y clínicas similares ${ }^{(1,2)}$. La EPI puede presentarse de forma asintomática; sin embargo, con el uso de la TACAR o mediante las pruebas de función pulmonar se puede hacer un diagnóstico temprano para instaurar un tratamiento oportuno y apropiado, teniendo en cuenta que estas entidades pueden progresar hasta fibrosis pulmonar a diferentes velocidades según la enfermedad de base ${ }^{(2)}$.

Dentro del grupo de la EPI podemos identificar distintos patrones radiológicos determinados según sus características y hallazgos en imagen, específica- 
mente. La neumonía intersticial no específica (NINE), la neumonía intersticial usual (NIU), la neumonía de organización (NO) y la neumonía intersticial linfoide (NIL) han sido descritas en estos pacientes (figura 1) (1). Los pacientes con enfermedades dermatológicas tienen una mayor prevalencia de presentación de EPI, con variaciones en la frecuencia de presentación y el patrón predominante de acuerdo con cada una de las entidades (tabla 1) ${ }^{(2-7)}$.

La importancia de esclarecer el patrón de la EPI radica en el factor pronóstico y la posibilidad de orientar hacia un tratamiento específico. Tal es el caso de la NO, que se caracteriza por una buena respuesta al manejo con corticosteroides (1). Otro patrón importante que debe resaltarse es la NINE, que es el patrón más frecuente en la EPI, especialmente frecuente en entidades como la esclerosis sistémica y la polimiositis/dermatomiositis (1). La NINE tiene dos fases de presentación: una fase celular, en donde se inicia el compromiso pulmonar, y una fase fibrótica, en donde llega a estar mucho más comprometida la arquitectura pulmonar con fibrosis; algunas veces es imposible diferenciar del patrón de NIU (1). El patrón de NIU es el más frecuente en artritis reumatoide (1).

Tabla 1. Compromiso intersticial en enfermedades dermatológicas.

\begin{tabular}{|c|c|c|c|c|c|}
\hline & $\begin{array}{l}\text { Esclerosis } \\
\text { sistémica }\end{array}$ & $\begin{array}{c}\text { Dermatomiositis } \\
\text { / polimiositis }\end{array}$ & $\begin{array}{l}\text { Artritis } \\
\text { reumatoide }\end{array}$ & $\begin{array}{l}\text { Lupus } \\
\text { eritematoso } \\
\text { sistémico }\end{array}$ & $\begin{array}{l}\text { Síndrome de } \\
\text { Sjögren }\end{array}$ \\
\hline $\begin{array}{l}\text { Prevalencia de } \\
\text { EPI }\end{array}$ & $45 \%$ & $20 \%$ a $50 \%$ & $20 \%$ a $30 \%$ & $2 \%$ a $8 \%$ & $50 \%$ \\
\hline \multicolumn{6}{|l|}{$\begin{array}{l}\text { Patrón } \\
\text { radiológico }\end{array}$} \\
\hline \multicolumn{6}{|l|}{$\begin{array}{l}\text { Neumonía } \\
\text { intersticial no } \\
\text { específica (NINE) }\end{array}$} \\
\hline \multicolumn{6}{|l|}{$\begin{array}{l}\text { Neumonía } \\
\text { intersticial usual } \\
\text { (NIU) }\end{array}$} \\
\hline \multicolumn{6}{|l|}{$\begin{array}{l}\text { Neumonía de } \\
\text { organización } \\
\text { (NO) }\end{array}$} \\
\hline $\begin{array}{l}\text { Neumonía } \\
\text { intersticial } \\
\text { linfoide (NIL) }\end{array}$ & & & & & \\
\hline
\end{tabular}



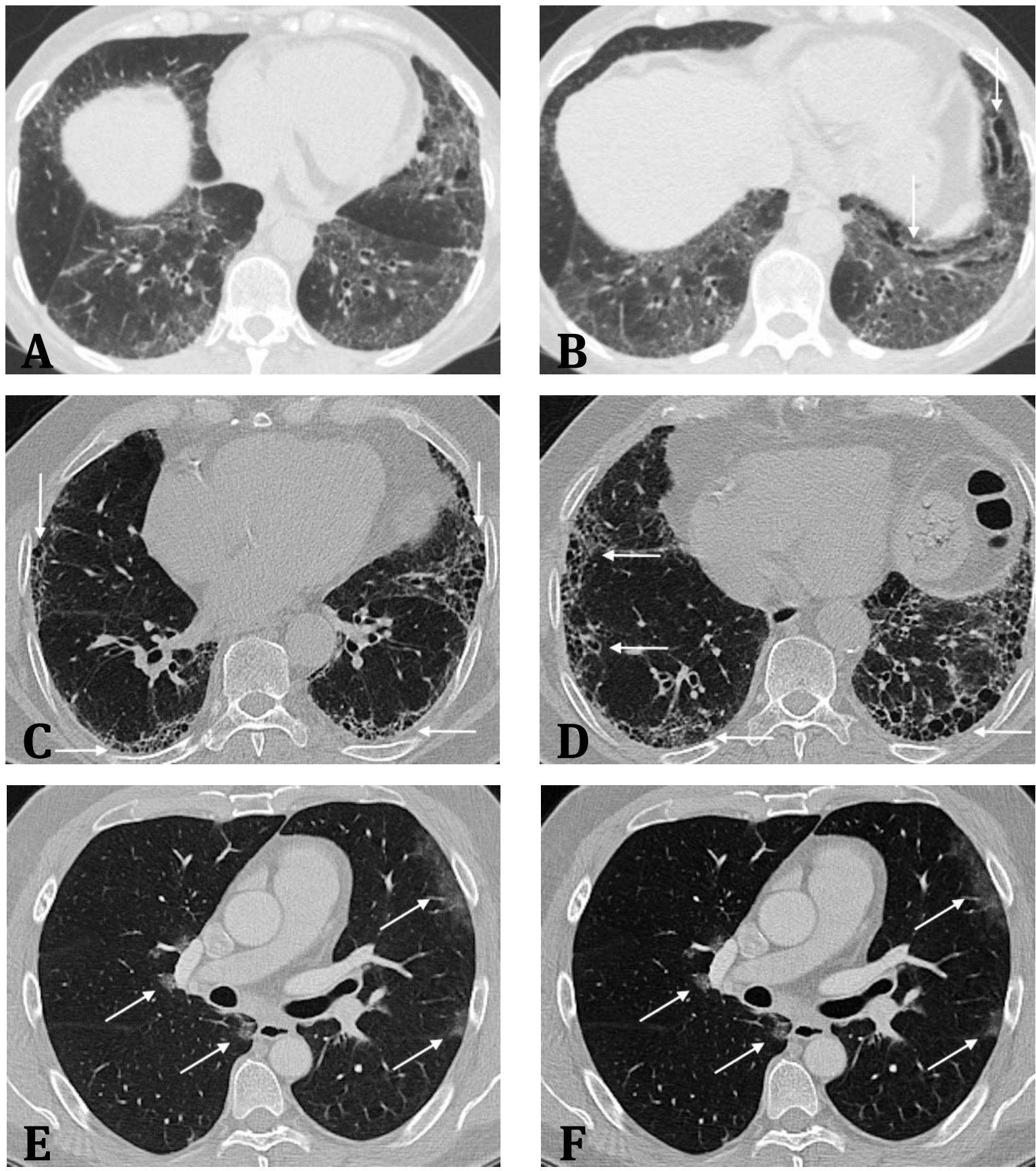

Figura 1. Tomografía de tórax, ventana para pulmón. A-B) Corte axial en lóbulos inferiores. Se observa alteración de la arquitectura pulmonar con presencia de opacidades en "vidrio esmerilado" asociada a reticulación y bronquiectasias por tracción (flechas) sin formación de "panal de abejas", lo que configura un patrón de neumonía intersticial no específica. C-D) Corte axial en lóbulos inferiores. Se observa alteración de la arquitectura pulmonar con áreas de panal de abejas de localización subpleural (flechas) asociada a engrosamiento irregular de los septos interlobulillares y bronquiectasias por tracción, que configuran un patrón de neumonía intersticial usual. E-F) Corte axial en los lóbulos inferiores. Se observan opacidades pseudonodulares en vidrio esmerilado de predominio subpleural, migratorias en controles posteriores, que corresponden a patrón de neumonía de organización. 


\section{NEUROFIBROMATOSIS TIPO 1}

La neurofibromatosis tipo 1 (NF-1) o enfermedad de Von Recklinghausen se cataloga como un síndrome neurocutáneo con patrón de herencia autosómico dominante por mutación del gen $\mathrm{NF} 1$, localizado en el cromosoma $17^{(8)}$. Sin embargo, en el 50\% de los casos puede ocurrir por una mutación espontánea ${ }^{(9)}$. La NF1 es la enfermedad más común del grupo de las facomatosis y se presenta en 1 de cada 2000 nacidos vivos ${ }^{(9)}$.

Las manifestaciones radiológicas torácicas son más frecuentes en los tejidos blandos y se relacionan con la distribución de los nervios periféricos en la superficie del tórax ${ }^{(8)}$. Dentro de los hallazgos más frecuentes se encuentra los neurofibromas subcutáneos bien definidos, la escoliosis torácica, las alteraciones en la morfología de los cuerpos vertebrales, el compromiso mediastinal por tumores neurogénicos y las anormalidades en la configuración de las costillas debidas a displasias óseas o a erosión secundaria a los neurofibromas adyacentes (figura 2) ${ }^{(9)}$. En el diagnóstico di- ferencial de los fibromas subcutáneos se deben considerar las neoplasias primarias y los quistes sebáceos o quistes epidérmicos vistos en el síndrome de Gardner (9). El compromiso pulmonar llega a ser poco común en la NF1, aunque en caso de estar presente, se evidenciarían bullas, de predominio en los lóbulos superiores, de ubicación subpleural bilateral y compromiso fibrótico intersticial ${ }^{(8)}$.

\section{ESCLEROSIS TUBEROSA}

La esclerosis tuberosa es una enfermedad autosómica dominante relacionada con la mutación del gen supresor de los tumores TSC1 en el cromosoma 9 y TSC2 en el cromosoma 16, genes de gran importancia en la codificación de hamartina y tuberina para la regulación de la proliferación y diferenciación celular ${ }^{(10)}$.

El compromiso torácico de esta entidad se observa principalmente por la linfangioleiomiomatosis (LAM), la cual afecta especialmente a las mujeres en edad re-
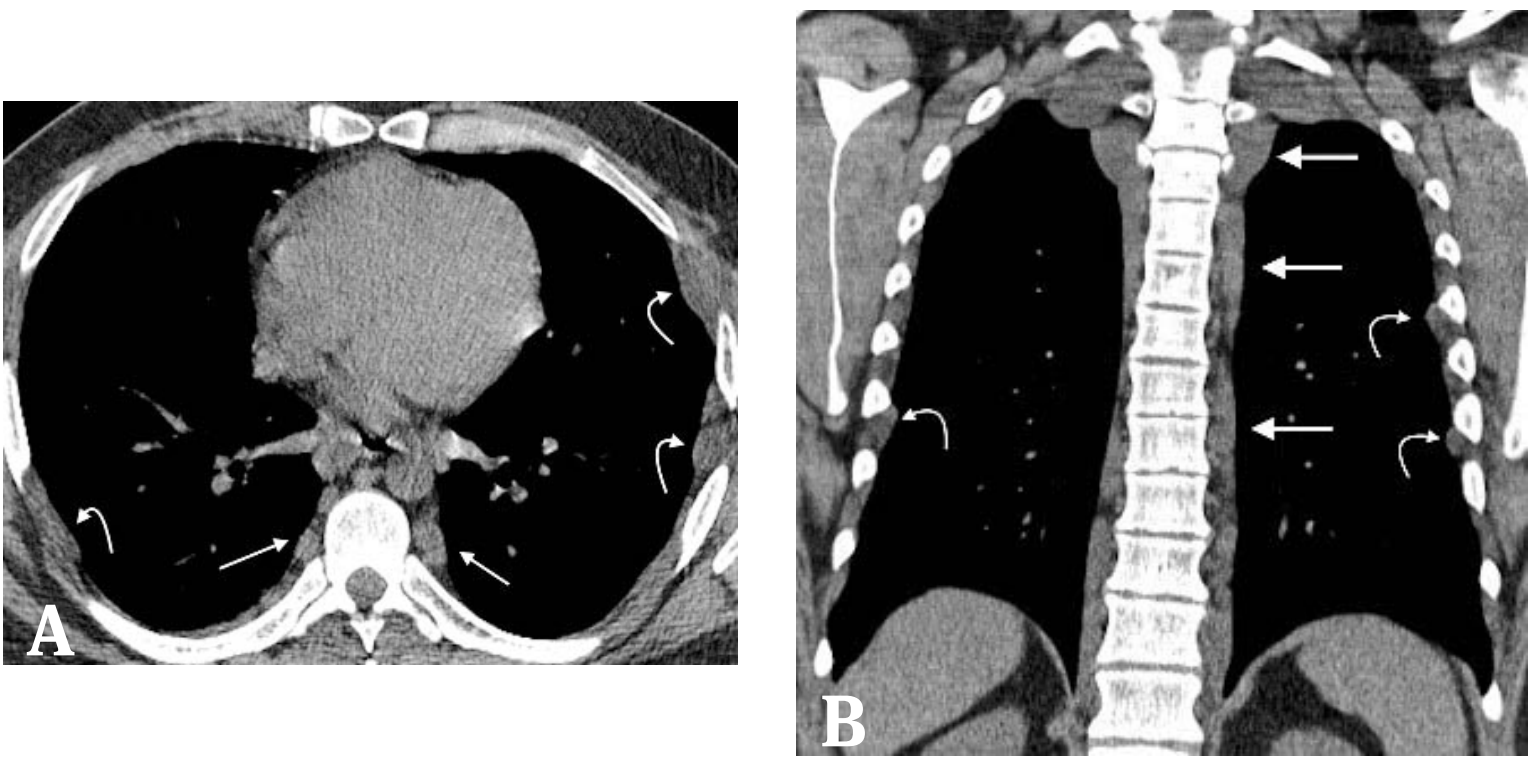

Figura 2. Paciente con antecedente de neurofibromatosis. Tomografía computarizada multidetectora simple de tórax en ventana mediastínica. A) Corte axial en el tercio medio del hemitórax. B) Reconstrucción coronal. Se observan nódulos sólidos ovalados y redondeados con densidad de tejidos blandos en el espacio mediastínico paravertebral (flechas rectas) y algunas en los espacios intercostales (flechas curvas) correspondientes a neurofibromas. 
productiva y muy esporádicamente a los hombres ${ }^{(10)}$. La LAM tiene una frecuencia estimada de $1 \%$ a $3 \%$ de los pacientes con esclerosis tuberosa ${ }^{(10,11)}$. Se caracteriza por un reemplazo quístico del parénquima pulmonar secundario a la proliferación de células de esclerosis múltiple, que son similares a las células de músculo liso alveolar, pero con receptores de progesterona, estrógenos y proteínas relacionadas con melanoma ${ }^{(12)}$. La radiografía de tórax es normal hasta en el $26 \%$ de los pacientes y en el porcentaje restante se encuentra hiperinsuflación pulmonar o algunas opacidades reticulares ${ }^{(13)}$. La TACAR es el método ideal para el diagnóstico, ya que permite evidenciar quistes pulmonares de paredes delgadas no mayores de $20 \mathrm{~mm}$, distribuidos de manera difusa en el parénquima pulmonar, y ocasionalmente algunos nódulos pulmonares difusos (figura 3). La presencia de nódulos de mayor tamaño debe hacer sospechar angiomiolipomas pulmonares en el diagnóstico diferencial ${ }^{(8)}$.

\section{SARCOIDOSIS}

La sarcoidosis se define como un proceso inflamatorio crónico con una etiología no clara, cuya principal característica es la formación de granulomas no caseificantes en múltiples órganos, siendo el pulmón uno de los órganos más comúnmente afectados ${ }^{(8)}$. Se describe que del 20\% al 35\% de los pacientes con diagnóstico de sarcoidosis van a presentar manifestaciones cutáneas en algún punto de su enfermedad ${ }^{(8)}$.
Usualmente los pacientes no presentan sintomatología respiratoria, o en caso de presentarla, puede llegar a ser muy inespecífica. El estudio inicial es una radiografía de tórax, que puede ser normal o presentar adenomegalias mediastínicas o parahiliares, nódulos pulmonares, masas o incluso, en estadios finales, cambios de fibrosis pulmonar ${ }^{(8)}$. La TCMD muestra adenomegalias mediastínicas y parahiliares bilaterales, que usualmente son simétricas en su forma, tamaño y realce con el medio de contraste (figura 4); algunas pueden presentar calcificaciones con patrón en "cáscara de huevo". Otros hallazgos que se pueden encontrar son micronódulos (<10 mm) de distribución perilinfática, los cuales pueden confluir formando conglomerados o masas. En enfermedad crónica pueden encontrarse cambios de fibrosis pulmonar, que suelen predominar en los lóbulos superiores con pérdida de volumen, bronquiectasias y reticulación ${ }^{(14)}$. Otros compromisos menos frecuentes en el tórax son las placas pleurales, la estenosis de la tráquea por compresión extrínseca secundaria a las adenomegalias mediastínicas y el engrosamiento de las paredes de los bronquios ${ }^{(15,16)}$.

\section{ARTRITIS REUMATOIDE}

La artritis reumatoide (AR) es una entidad que se caracteriza por tratarse de una poliartropatía inflamatoria, simétrica, con especial compromiso de manos y pies; sin embargo, puede tener compromiso en otros órganos, en especial entidades como la pericarditis, la
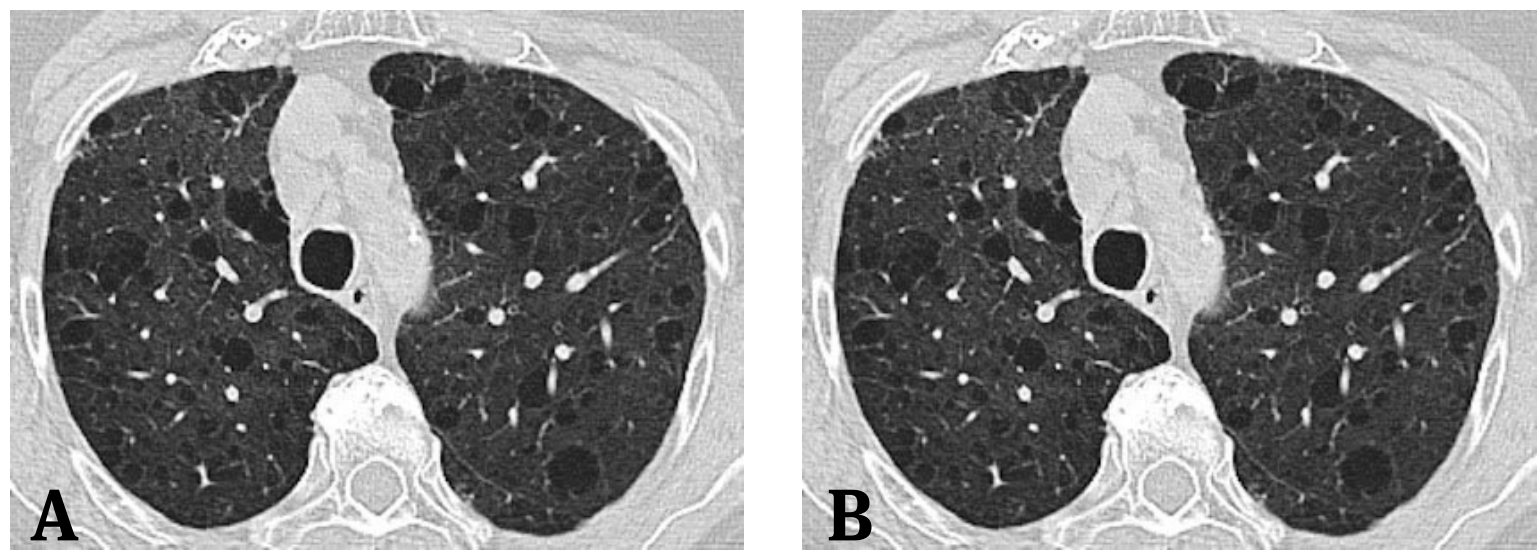

Figura 3. Paciente con antecedente conocido de esclerosis tuberosa. Tomografía de tórax, ventana para pulmón. A-B) Corte axial en lóbulos superiores. Múltiples áreas quísticas de paredes delgadas en los diferentes segmentos pulmonares no mayores de $20 \mathrm{~mm}$ en relación con linfangioleiomiomatosis. 

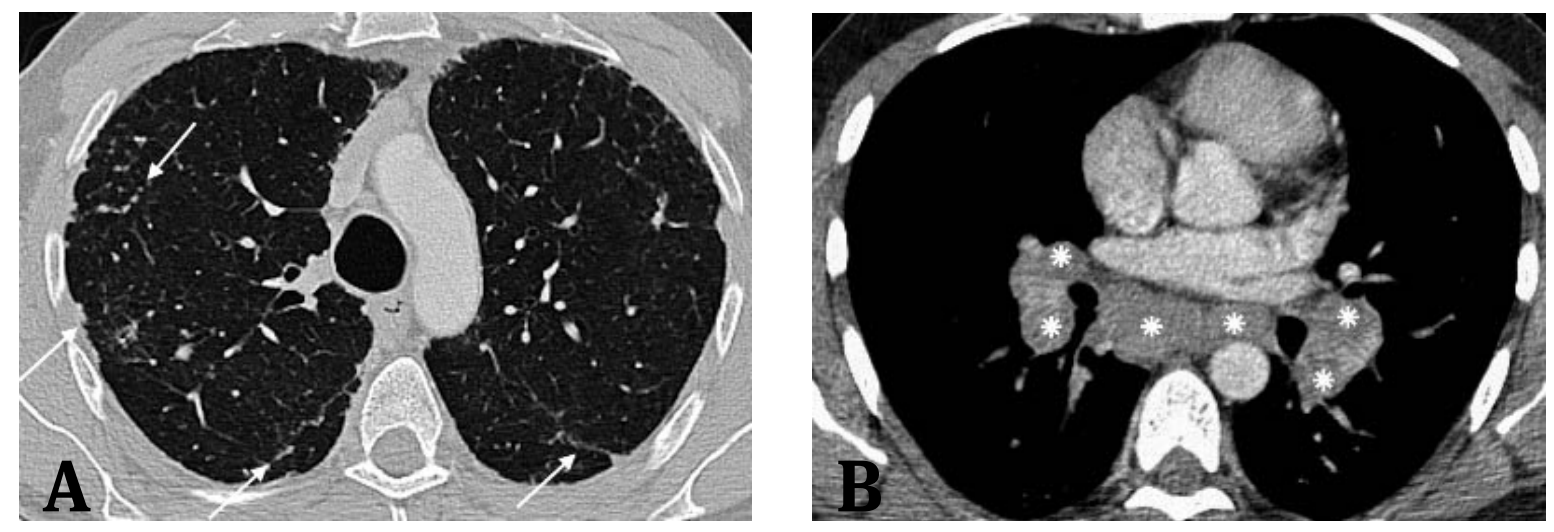

Figura 4. A) Tomografía simple de tórax, ventana para pulmón en lóbulos superiores. Se observan nódulos sólidos con densidad de tejidos blandos no mayores de $5 \mathrm{~mm}$, de disposición perilinfática, más evidentes en las cisuras con apariencia en "rosario" (flechas). B) Tomografía con contraste de tórax, ventana para mediastino a nivel de la arteria pulmonar. Múltiples adenomegalias mediastínicas de predominio parahiliar bilateral, simétricas y bien definidas, que demuestran realce homogéneo posterior a la administración del medio de contraste (asteriscos).

esplenomegalia, la inflamación ocular y los nódulos subcutáneos ${ }^{(17)}$. Dichas manifestaciones extraarticulares están presentes en un $40 \%$ de los diagnosticados con AR y son más graves en pacientes fumadores ${ }^{(17-19)}$.

El hallazgo torácico más frecuente en la AR es el compromiso pleural, que está dado por el engrosamiento o el derrame pleural, evidente en el $40 \%$ al $70 \%$ de los pacientes ${ }^{(1)}$. Suele estar relacionado con enfermedad articular activa de largo tiempo de evolución ${ }^{(1)}$. Este tipo de compromiso crónico puede llevar a un efecto restrictivo pulmonar por un engrosamiento pleural grave, que condiciona una pérdida de volumen del hemitórax afectado ${ }^{(17,20)}$.

El compromiso pulmonar por EPI afecta del 20\% al $30 \%$ de los pacientes y muestra patrones de NIU, NINE y NO, siendo el patrón predominante la NIU ${ }^{(17)}$. También podemos encontrar neumonía intersticial linfocítica (NIL) en la variedad del compromiso pulmonar en pacientes con AR. En la TACAR, la NIL se caracteriza por la presencia de nódulos centrolobulillares con halo en vidrio esmerilado; algunos de estos configuran un patrón en “árbol en gemación”, opacidades en vidrio esmerilado en el parénquima pulmonar y bronquiectasias ${ }^{(17)}$.

Los nódulos pulmonares, también conocidos como nódulos reumatoides (figura 5), se evidencian en la TACAR aproximadamente en el $20 \%$ de los pacientes con AR y son más frecuentes en el género masculino ${ }^{(21)}$. Estos nódulos son evidentes durante los estadios avanzados de la enfermedad; sin embargo, no reflejan la actividad de la enfermedad, aunque están relacionados con la presencia de nódulos subcutáneos ${ }^{(17)}$. Suelen estar localizados en la periferia del parénquima pulmonar y predominan en los lóbulos superiores y medios ${ }^{(1)}$. Cuando estos nódulos se calcifican, se debe considerar la presencia de síndrome de Caplan, usualmente relacionado con enfermedad ocupacional, siendo la más frecuente la silicosis ${ }^{(1)}$. La bronquiolitis obliterante es caracterizada como un patrón en "mosaico de atenuación” con característico atrapamiento aéreo en las imágenes obtenidas en espiración, que representa la enfermedad de la vía aérea pequeña ${ }^{(1)}$. Otra complicación descrita es la bronquiolitis folicular, también llamada hiperplasia pulmonar linfoide, que se encuentra dentro del espectro de la NIL ${ }^{(17)}$. En la TACAR, esta se evidencia como nódulos de distribución centrilobulillar, algunos de ellos con halo de vidrio esmerilado ${ }^{(17)}$.

El compromiso de la vía aérea es el segundo tipo de compromiso reportado en frecuencia en la AR. Está dado principalmente por la presencia de bronquiectasias no relacionadas con fibrosis pulmonar y afecta aproximadamente al 30\% de los pacientes ${ }^{(17,22)}$. Además, se puede evidenciar un compromiso de la vía aérea superior por artritis cricoaritenoidea o incluso parálisis de las cuerdas vocales ${ }^{(17,22)}$. 

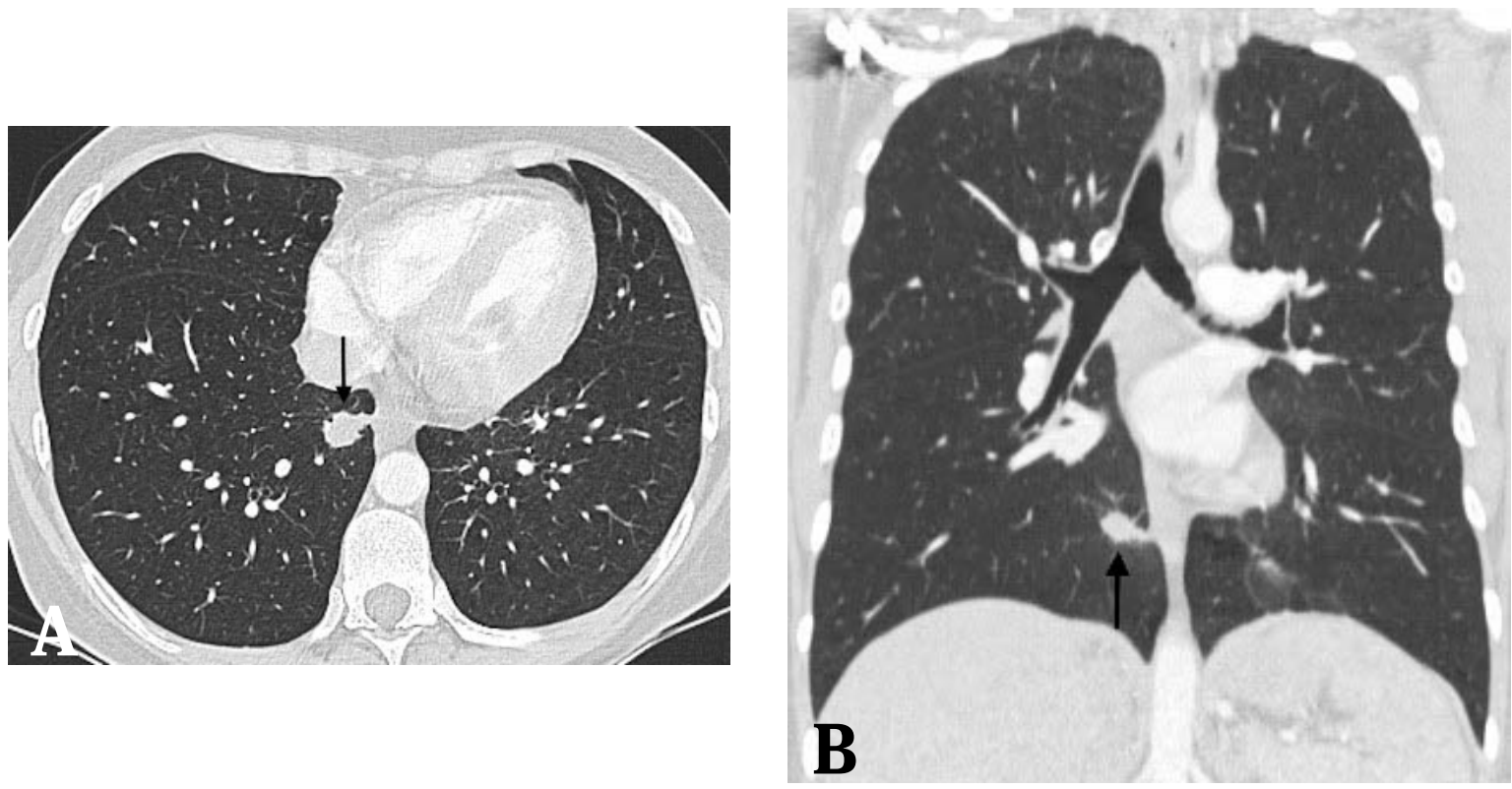

Figura 5. A)Tomografía de tórax, ventana para pulmón, corte axial en los lóbulos inferiores. B) Reconstrucción coronal. Nódulo sólido, de localización subpleural, de morfología ovalada, con densidad de tejidos blandos, que demuestra contornos parcialmente definidos (flecha) con discreto halo en vidrio esmerilado relacionado con nódulo reumatoideo, que resolvió en controles sucesivos.

Por último, se deben tener en cuenta los compromisos secundarios al tratamiento de la AR, donde sobresale el compromiso pulmonar por metotrexato, que tiene una frecuencia de toxicidad pulmonar del 5 al 10\% de los casos; sin embargo, no se ha encontrado relación entre la dosis o el tiempo de duración para desarrollar toxicidad pulmonar ${ }^{(23)}$. Vale la pena también mencionar la reactivación de la tuberculosis pulmonar con el uso de antagonistas del factor de necrosis tumoral (FNT) ${ }^{(24,25)}$.

\section{POLIMIOSITIS/ \\ DERMATOMIOSITIS}

La polimiositis y la dermatomiositis son enfermedades del tejido conectivo, caracterizadas por un proceso inflamatorio del músculo estriado esquelético, que lleva a pérdida progresiva de la fuerza en los grupos musculares proximales, además de mialgias y elevación de la creatina-cinasa (CK) ${ }^{(26)}$. Si el compromiso muscular se asocia a erupción cutánea, se denominaría dermatomiositis cutánea, representada en las clásicas pápulas de Gottron ${ }^{(8)}$. La etiología es poco clara, pero se cree que la polimiositis está relacionada con antí- genos musculares y la dermatomiositis con daño de la microvasculatura mediado por una respuesta inmunitaria que lleva a hipoperfusión y presencia de células inflamatorias en la región perifascicular ${ }^{(8,26)}$.

En las manifestaciones torácicas de estas dos entidades se describen neumonías intersticiales, vasculitis, hipertensión pulmonar y atrofia de los músculos torácicos ${ }^{(8)}$. El compromiso pulmonar intersticial está mediado por el anticuerpo asociado a miositis y los anticuerpos contra la enzima aminoacil ARNt sintetasa, que se correlacionan con su presentación clínica y el riesgo de desarrollar una enfermedad intersticial pulmonar, usualmente representada por la neumonía de organización (NO), que pueden presentarse incluso antes del compromiso cutáneo ${ }^{(1,8,27)}$. Otro patrón de EPI reportado es la NINE; sin embargo, se debe aclarar que la NINE y la NO no son mutuamente excluyentes y se pueden obtener hallazgos radiológicos e histológicos de ambas entidades simultáneamente en un mismo paciente ${ }^{(8)}$. Otro tipo de compromiso son los cambios secundarios al tratamiento, sobreinfección, neumonía por inmunosupresión, neumonía por aspiración relacionada con atrofia de los músculos de la faringe, falla 
respiratoria por adelgazamiento de los músculos intercostales y efectos tóxicos del tratamiento farmacológico; este último también puede derivar en un patrón de $\mathrm{NO}^{(8)}$.

\section{ESCLEROSIS SISTÉMICA}

La esclerosis sistémica, también conocida como esclerodermia, es una patología autoinmunitaria caracterizada por producir fibrosis, daño vascular e inflamación (1). Se describe cómo al inicio de la enfermedad solo el $1 \%$ de los pacientes tiene síntomas respiratorios, porcentaje que aumenta a 60\% durante el trascurso de la enfermedad $^{(20,28)}$.

La fibrosis pulmonar puede estar presente entre un $10 \%$ y un $16 \%$ de los pacientes con esclerosis sistémica difusa, la cual guarda relación con anticuerpos antitopoisomerasa ${ }^{(20,29)}$. La NINE es el patrón radiológico de compromiso intersticial más frecuente ${ }^{(8)}$. Un compromiso que supere el $20 \%$ se considera extenso y lleva una mayor tasa de mortalidad y a un rápido deterioro de la función pulmonar ${ }^{(1)}$. Otros hallazgos encontrados son la hipertensión pulmonar arterial, que guarda relación con la presencia de anticuerpos anticentrómeros (ACA) ${ }^{(8,29)}$ y probablemente esté relacionada con enfermedad del tejido conectivo. Una manifestación muy frecuente, presente en el $97 \%$ de los pacientes, es la dilatación esofágica, la cual puede predisponer a neumonía por aspiración o bronquiolitis (figura 6) ${ }^{(1)}$.

\section{SÍNDROME DE SJÖGREN}

El síndrome de Sjögren (SJ) es considerado una enfermedad inflamatoria autoinmunitaria relacionada con la infiltración por parte de linfocitos $\mathrm{T}$ en distintos órganos, siendo los más comúnmente afectados las glándulas exocrinas (lacrimales y salivares), que lleva a ausencia en la secreción glandular ${ }^{(7,30)}$. En la anamnesis se debe preguntar por los denominados síntomas $B$, ya que los pacientes con SJ tienen más riesgo de desarrollar linfoma, usualmente linfoma no-Hodgkin de células $B^{(1)}$.

El 50\% de los casos de SJ presenta EPI (2). Dicho compromiso es más común en pacientes con SJ primario y se manifiesta con patrones de NINE, NIU, NO y NIL, menos común con patrones de bronquiolitis o incluso amiloidosis $^{(1)}$. En la TCMD de la NIL se describen áreas de vidrio esmerilado, engrosamiento de los septos interlobulillares, nódulos y quistes de pared delgada (figura 7) ${ }^{(1)}$. Ya en la bronquiolitis folicular, en la TCMD se evidencian quistes, que se relacionan por la infiltración de linfocitos en la pared, que producen una dilatación bronquiolar secundaria ${ }^{(20)}$. Otros compromisos evidenciados son el engrosamiento de las paredes
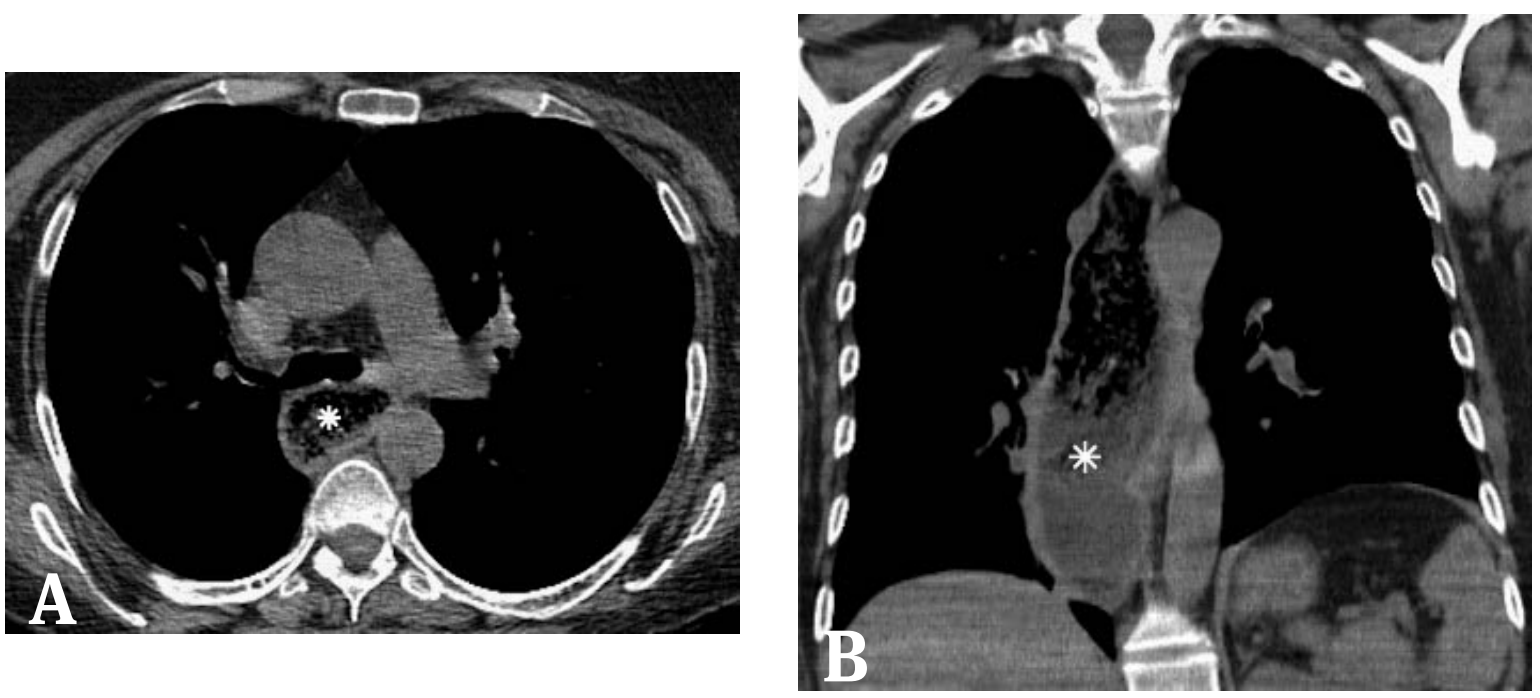

Figura 6. Paciente con antecedente de esclerosis sistémica. Tomografía de tórax, ventana para mediastino. A) Corte axial en lóbulos superiores. B) Reconstrucción coronal. Hay dilatación del esófago en sus tres tercios, que demuestra contenido alimentario en su interior con burbujas aéreas y apariencia heterogénea (asteriscos). 

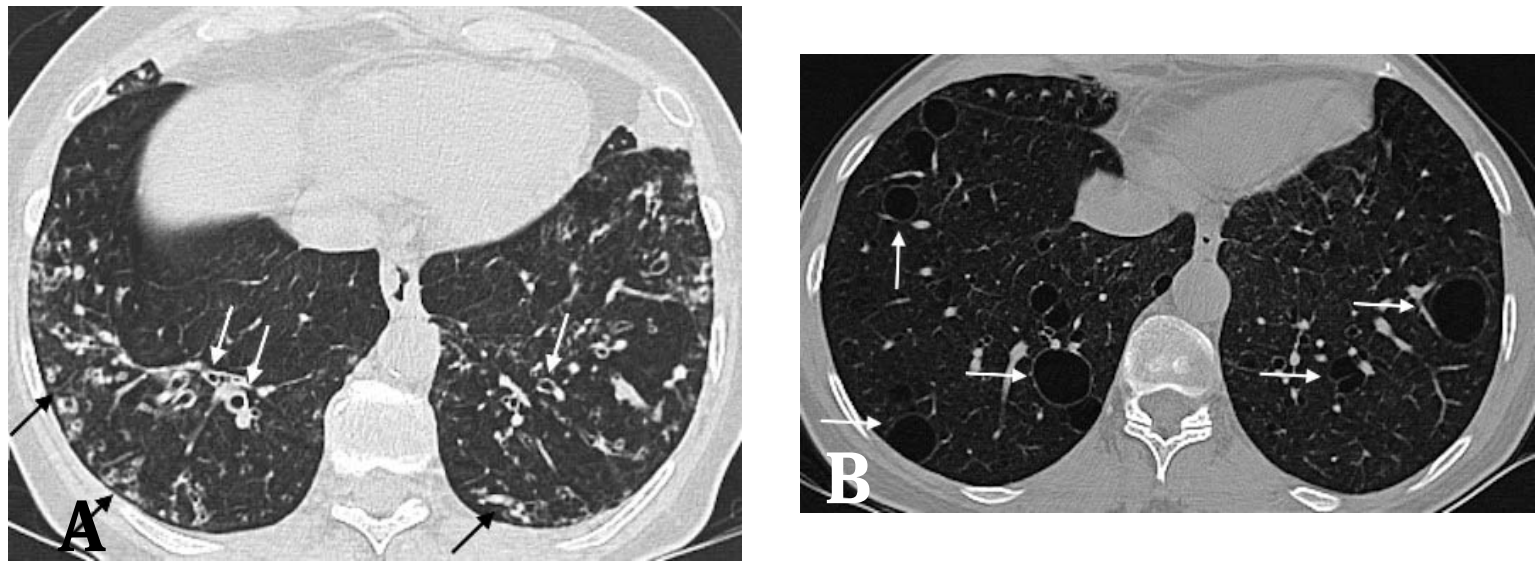

Figura 7. A)Tomografía computarizada, ventana para pulmón, corte axial en lóbulos inferiores. Presencia de bronquiectasias (flecha blanca) en los lóbulos inferiores, con engrosamiento de las paredes bronquiales y áreas de árbol en gemación (flecha negra), especialmente de disposición subpleural como representación de bronquiolitis asociada o impactación de moco, en paciente con antecedente de síndrome de Sjögren. B) Tomografía computarizada, ventana para pulmón, corte axial en los lóbulos inferiores. Imágenes quísticas, de pared delgada (flechas blancas), algunas de ellas con nódulo en la pared de la lesión; tienen predominio en los lóbulos inferiores en un paciente con diagnóstico de neumonía intersticial linfoide.

\section{Puntos clave}

- Etiologías infecciosas, inflamatorias, enfermedades del tejido conectivo, entidades hereditarias o adquiridas pueden tener compromiso en ambos órganos durante el curso de la enfermedad.

- La importancia de esclarecer el patrón de EPI es el factor pronóstico y la posibilidad de orientar hacia un tratamiento específico.

- El manejo multidisciplinario que incluya el abordaje radiológico en relación con las manifestaciones cutáneas garantiza enormes posibilidades diagnósticas y puede ayudar a la toma de decisiones clínicas apropiadas y oportunas.

bronquiales, las bronquiectasias, las broquioloectasias y las áreas de atrapamiento aéreo (figura 7) ${ }^{(20)}$. Por último, si el radiólogo describe consolidaciones, nódulos de gran tamaño y derrame pleural en la TCMD, se debe descartar la presencia de linfoma ${ }^{(20)}$.

\section{LUPUS ERITEMATOSO SISTÉMICO}

El lupus eritematoso sistémico (LES) es una enfermedad autoinmunitaria multisistémica del tejido conectivo, que afecta la piel, las articulaciones, los riñones y el sistema nervioso central ${ }^{(8)}$. El compromiso cutáneo ocurre en el 75\% de los casos durante el trascurso de la enfermedad, siendo fundamental su caracterización temprana debido a que el 25\% de los pacientes presentan alteraciones en la piel semanas o meses previos a exhibir los síntomas sistémicos ${ }^{\left({ }^{31}\right)}$.

Es importante precisar cómo la sintomatología respiratoria en lupus es nula o leve; sin embargo, los pacientes sí pueden consultar por dolor pleurítico como síntoma principal, sintomatología que guarda relación con la evidencia de derrame pleural bilateral y engrosamiento pleural relacionado con la serositis característica ${ }^{(8)}$. Estos hallazgos se reportan en el 61\% de los pacientes y es importante descartar las complicaciones por pleuritis refractaria, como el fibrotórax, que se puede visualizar en la TCMD como derrame pleural no libre o colecciones pleurales, el engrosamiento pleural liso y las atelectasias subyacentes ${ }^{(20,32)}$. Otras 

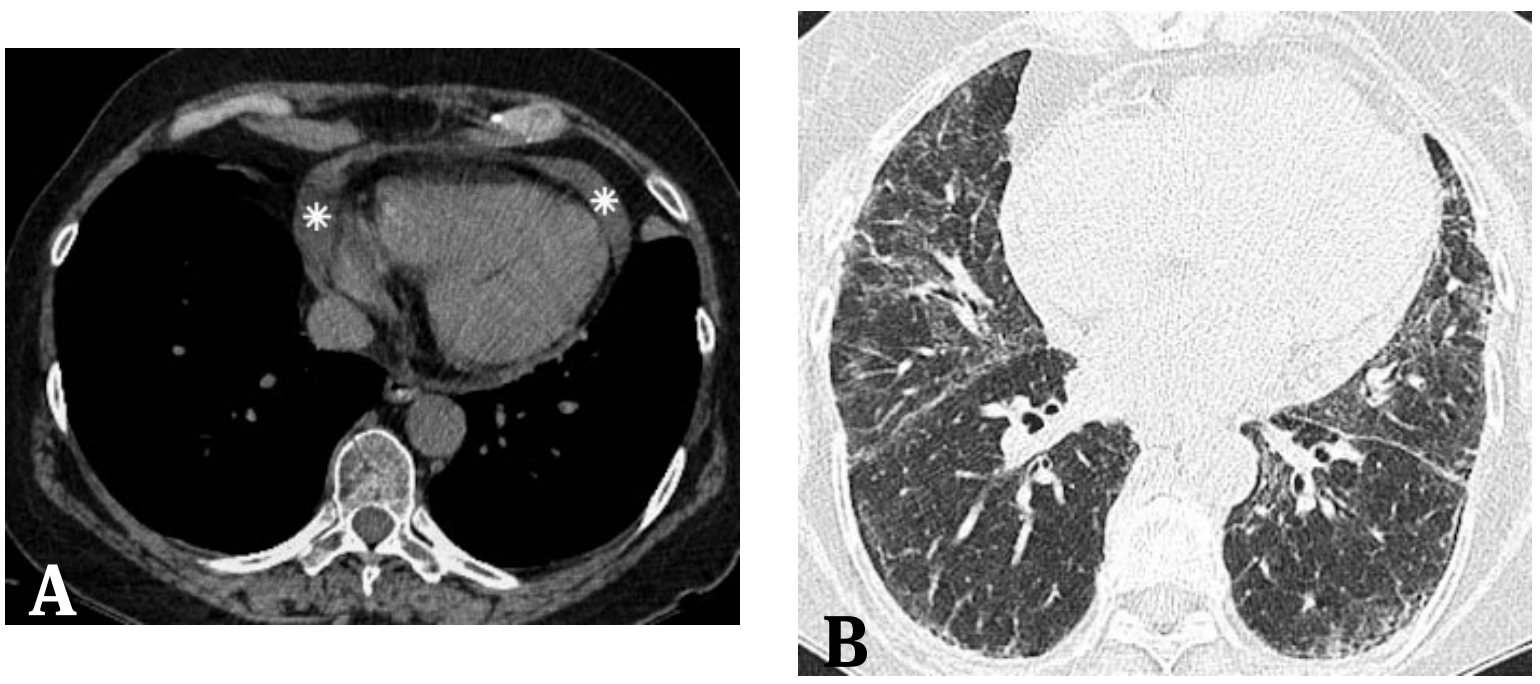

Figura 7. Paciente con diagnóstico de lupus eritematoso sistémico. A) Tomografía computarizada en ventana mediastínica. Se aprecia hipodensidad que rodea al corazón (asteriscos) correspondiente a líquido por derrame pericárdico en un paciente con diagnóstico de pericarditis por serositis en el contexto de LES. B) Tomografía computarizada de alta resolución, ventana para pulmón, corte axial en los lóbulos inferiores. Alteración de la arquitectura pulmonar con opacidades en vidrio esmerilado asociada a reticulación y escasas bronquiectasias sin formación de panal de abeja, que configura un patrón de neumonía intersticial no específica.

manifestaciones en el tórax son los hallazgos de neumonía adquirida en la comunidad, donde también se debe pensar en microorganismos atípicos debido a las alteraciones (primarias o secundarias) del sistema inmunitario de estos pacientes, especialmente aquellos en manejo con inmunomodulador ${ }^{(20)}$.

En cuanto al compromiso pulmonar intersticial en el LES, este tiende a ser poco común y afecta entre el $2 \%$ y el $8 \%$ de los pacientes ${ }^{(2,33)}$. La presentación más común es la NINE (figura 8) ${ }^{(1,20)}$, donde la radiografía de tórax tiende a ser poco significativa en estadios leves a moderados ${ }^{\left({ }^{2} 2\right)}$, por la ausencia de hallazgos radiográficos, a diferencia de la TACAR, que es de gran importancia para detectar el compromiso intersticial temprano ${ }^{(33)}$.

Otras entidades que deben descartarse son las relacionadas con el compromiso cardiovascular y la serositis, presentes en el 58\% a 77\% de los casos, siendo la presentación más frecuente la pericarditis, que se manifestará como derrame pericárdico (figura 8) ${ }^{(32)}$.

\section{PSORIASIS}

La psoriasis en una enfermedad inflamatoria crónica que afecta del $2 \%$ al 3\% de la población general ${ }^{(34)}$.
Múltiples estudios han demostrado que los pacientes con psoriasis y particularmente aquellos con formas graves de la enfermedad tienen una mayor incidencia de infarto de miocardio, accidente cerebrovascular, diabetes y mortalidad cardiovascular ${ }^{\left({ }^{35}\right)}$, que se produce por la carga inflamatoria de la enfermedad y no por la enfermedad. También se ha demostrado su asociación a síndrome metabólico, enfermedad pulmonar obstructiva crónica (EPOC), asma, úlcera péptica, enfermedad hepática, insuficiencia renal y artritis reumatoide ${ }^{(36,37)}$.

En los últimos años se han publicado algunos estudios que demuestran la asociación de la psoriasis con diferentes patologías pulmonares tales como EPOC, hipertensión pulmonar y sarcoidosis ${ }^{\left({ }^{6}, 38\right)}$. No es clara la asociación de la EPOC a la psoriasis. Existen dos hipótesis: la primera, un estado inflamatorio crónico similar genera las dos enfermedades. Las respuestas inmunitarias mediadas por Th1 y Th17 incrementan los niveles de ciertas citocinas, como la interleucina 1 (IL1), IL-6, IL-8, y el FNT- $\alpha$. El incremento de los niveles de proteína C-reactiva (un biomarcador de inflamación sistémica) y citocinas proinflamatorias está asociado a EPOC. Se ha demostrado la asociación de IL-17 tanto en psoriasis como en EPOC ${ }^{(38)}$. El estudio realizado en población de Taiwán, el cual pretendía mostrar la aso- 
ciación entre psoriasis y EPOC, demostró que los pacientes con psoriasis tienen más riesgo de desarrollar EPOC en comparación con el grupo control $(p=0,001)$ (39).

Los pacientes con psoriasis tienen mayor riesgo de desarrollar EPI en comparación con la población general, siendo la fibrosis pulmonar idiopática la manifestación clínica más frecuente y el patrón de neumonía intersticial usual la manifestación radiológica más común ${ }^{35}$ ${ }^{40)}$. Otra interesante relación de la psoriasis con la patología pulmonar son los estudios que han explorado la asociación de sarcoidosis con psoriasis ${ }^{(41)}$. Se cree que ambas enfermedades comparten una vía fisiopatológica común relacionada con la activación de linfocitos en las vías Th1 y Th17 ${ }^{(42)}$.

\section{SARCOMA DE KAPOSI}

El sarcoma de Kaposi se define como lesiones mesenquimales multisistémicas que involucran los vasos sanguíneos y los vasos linfáticos ${ }^{(8)}$. El órgano más afectado es la piel; sin embargo, se deben tener en cuenta otros órganos comprometidos como los son el tracto gastrointestinal, los ganglios linfáticos y el pulmón ${ }^{(8)}$.

Se describe que el compromiso pulmonar está presente en un $45 \%$ de los casos, $15 \%$ de ellos sin compromiso cutáneo asociado ${ }^{(43)}$. El compromiso torácico puede involucrar el pulmón, la tráquea, la pleura o la pared torácica ${ }^{(8,43)}$. El estudio inicial con radiografía de tórax puede mostrar el compromiso por la enfermedad primaria o por infecciones por gérmenes oportunistas ${ }^{(43)}$. La radiografía de tórax puede ser una buena aproximación diagnóstica a la enfermedad primaria por sarcoma de Kaposi ${ }^{(43)}$ : muestra opacidades reticulares asociadas a nódulos pulmonares y engrosamiento broncovascular ${ }^{(8,44)}$. Es precisamente en esta situación en la cual la TACAR tiene indicación para evidenciar nódulos pulmonares de distribución peribroncovascular (figura 9) y adenomegalias en el 50\% de los casos $(8,43)$. Además, no se debe dejar de lado el compromiso de la pared torácica, donde el radiólogo puede reportar masas cutáneas y subcutáneas o lesiones líticas del esternón o en la columna torácica ${ }^{(43,45)}$.

\section{ENDOCARDITIS BACTERIANA}

La endocarditis bacteriana es la infección de las válvulas y las cavidades cardíacas que se desarrolla en pacientes con factores predisponentes ${ }^{(8)}$. El microorganismo causante más común es el Staphylococcus aureus ${ }^{(46)}$. Las manifestaciones cutáneas tienen una prevalencia de aproximadamente un $11,9 \%$, siendo más frecuentemente reportados los nódulos de Osler, las lesiones de Janeway y la hemorragia subungueal ${ }^{(8,47)}$.

En la radiografía de tórax, la TACAR y la TCMD es característica la aparición de múltiples nódulos pulmonares de márgenes mal definidos, de predominio

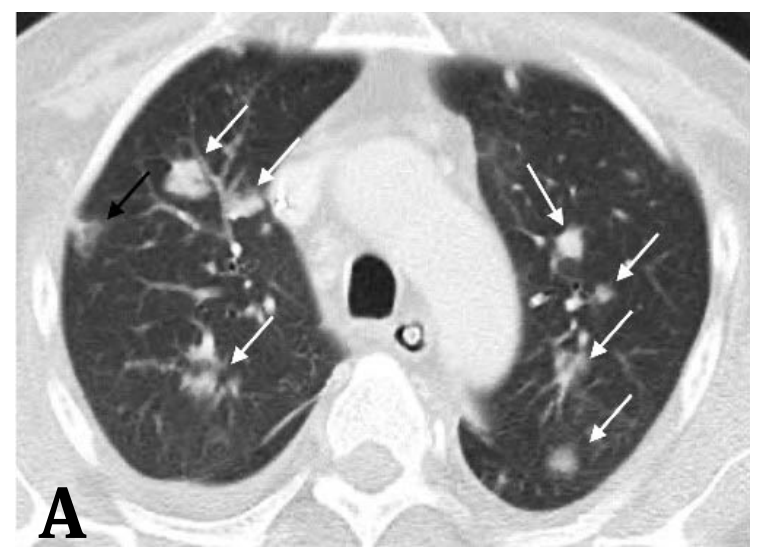

Figura 9. Tomografía computarizada, corte axial, en ventana de pulmón. Opacidades nodulares con densidad de tejido blando (flechas blancas) y algunas en vidrio esmerilado (flechas negras), de contornos parcialmente definidos, distribuidas en los diferentes segmentos pulmonares en un paciente con diagnóstico conocido de sarcoma de Kaposi en el contexto de infección por virus de inmunodeficiencia humana. 

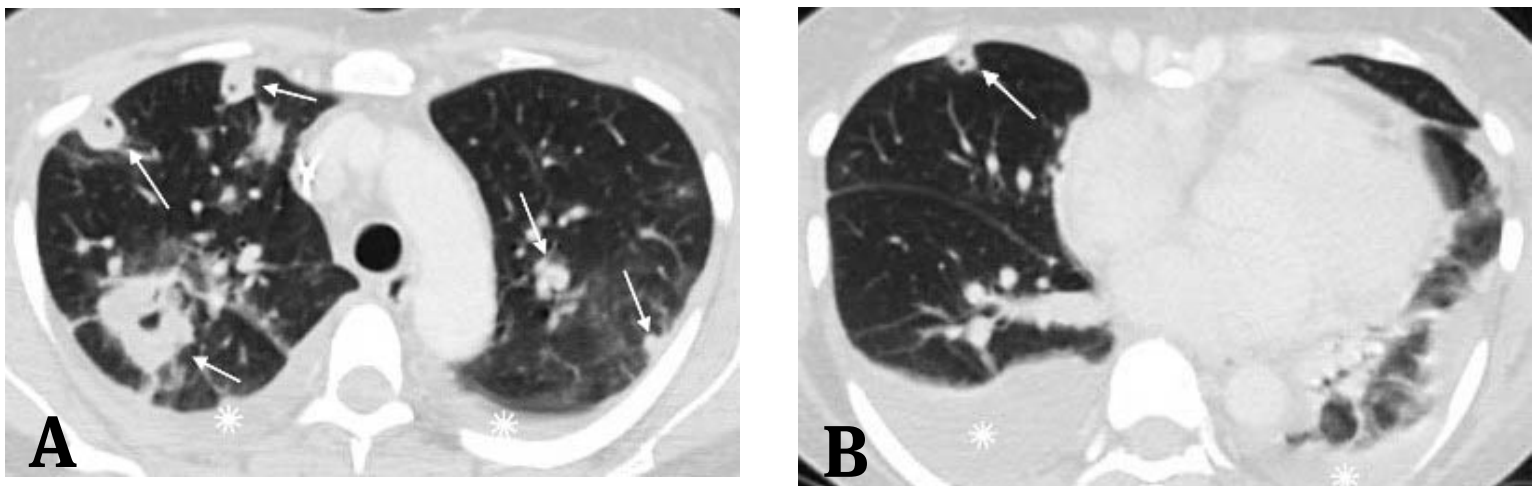

Figura 10. Tomografía de tórax, ventana para pulmón en los lóbulos superiores (A) y los lóbulos inferiores (B). Se observan múltiples nódulos sólidos, con densidad de tejido blando de predominio subpleural, en los diferentes segmentos (flechas blancas); algunos de los de mayor tamaño en el lóbulo superior derecho demuestran áreas de hipodensidad centrales relacionadas con cambios por cavitación. Hay derrame pleural bilateral que predomina en el lóbulo inferior derecho (asteriscos).

periférico, algunos de ellos cavitados y donde es importante indagar, con ayuda del radiólogo, por la presencia de vaso nutricio, hallazgo que orienta hacia un embolismo séptico (figura 10) ${ }^{(8,47)}$.

\section{SÍNDROME SAPHO}

El acrónimo SAPHO hace referencia a la aparición de sinovitis, hiperostosis, osteítis y lesiones de piel tales como pustulosis y acné grave ${ }^{(48)}$.

Dentro del estudio radiológico es importante tener claro que los hallazgos varían de acuerdo con la etapa de la lesión, con compromiso osteolítico en las etapas tempranas de la enfermedad y osteoproliferativo, en las etapas tardías ${ }^{(48)}$. En relación con la hiperostosis y la osteítis, se caracterizan por un incremento en la esclerosis del hueso, que usualmente se suele concentrar en huesos largos cuando afecta a niños y adolescentes ${ }^{(48)}$. En adultos, el compromiso principal es en la pared anterior del tórax, descrito como entesopatía costoclavicular y focos hiperostóticos localizados en el extremo proximal del esternón, adyacente a la primera costilla, hallazgos que se consideran decisivos en el diagnóstico temprano de la enfermedad ${ }^{(48,49)}$. Otra ayuda diagnóstica descrita es la gammagrafía ósea, que permite una detección de las lesiones en pacientes asintomáticos con evidencia de captación del radiotrazador, especialmente en la región esternoclavicular ${ }^{(49)}$.

\section{CONCLUSIONES}

El manejo multidisciplinario que incluya el abordaje radiológico en relación con las manifestaciones cutáneas garantiza enormes posibilidades diagnósticas y puede ayudar a la toma de decisiones clínicas apropiadas y oportunas, especialmente en el grupo de pacientes con enfermedades inmunológicas, en donde la ventana terapéutica es estrecha y las complicaciones son devastadoras. El advenimiento de los nuevos métodos diagnósticos acerca, cada vez más, al radiólogo con el dermatólogo y ofrece ilimitadas posibilidades para mejorar la calidad de vida de nuestros pacientes.

\section{REFERENCIAS}

1. Aluja-Jaramillo F, Acosta L, Gutiérrez FR. Neumonitis por hipersensibilidad y enfermedades del tejido conectivo. En: Avances en diagnóstico por imágenes: Tórax 2. Criales-Cortes JL, Gutiérrez FR. 2.a edición. Buenos Aires, Argentina: Ediciones Journal; 2019. p. 29-43.

2. Castelino FV, Varga J. Interstitial lung disease in connective tissue diseases: evolving concepts of pathogenesis and management. Arthritis Res Ther. 2010;12(4):213. doi: 10.1186/ar3097

3. Kim DS, Yoo B, Lee JS, Kim EK, Lim C, Lee S, et al. The major histopathologic pattern of pulmonary fibrosis in scleroderma is nonspecific interstitial pneumonia. Sarcoidosis Vasc Diffuse Lung Dis. 2002;19(2):121-7. PMID: 12108451 
4. De Lauretis A, Veeraraghavan S, Renzoni E. Review series: Aspects of interstitial lung disease: connective tissue disease-associated interstitial lung disease: how does it differ from IPF? How should the clinical approach differ? Chron Respir Dis. 2011;8(1):53-82. doi: 10.1177/1479972310393758

5. Kang EH, Lee EB, Shin KC, Im C, Chung D, Han S, et al. Interstitial lung disease in patients with polymyositis, dermatomyositis and amyopathic dermatomyositis. Rheumatology (Oxford). 2005;44(10):1282-6. doi: 10.1093/rheumatology/keh723

6. Tanaka N, Kim JS, Newell JD, Brown KK, Cool CD, Meehan R, et al. Rheumatoid arthritis-related lung diseases: CT findings. Radiology. 2004,232(1):8191. doi: 10.1148/radiol.2321030174

7. Ito I, Nagai S, Kitaichi M, Nicholson AG, Johkoh T, Noma S, et al. Pulmonary manifestations of primary Sjogren's syndrome: a clinical, radiologic, and pathologic study. Am J Respir Crit Care Med. 2005;171(6):632-8. doi: 10.1164/rccm.200403-4170C

8. Kanne JP, Yandow DR, Haemel AK, Mayer CA. Beyond skin deep: Thoracic manifestations of systemic disorders affecting the skin. Radiographics. 2011;31(6):1651-68. doi: 10.1148/rg.316115516

9. Fortman BJ, Kuszyk BS, Urban BA, Fishman EK. Neurofibromatosis Type 1: A Diagnostic Mimicker at CT. RadioGraphics. 2001;21(3):601-12. doi: 10.1148/radiographics.21.3. go1mao5601

10. Curatolo P, Bombardieri R, Jozwiak S. Tuberous sclerosis. The Lancet. 2008;372(9639):657-68. doi: 10.1016/s0140-6736(o8)61279-9

11. Hancock E, Osborne J. Lymphangioleiomyomatosis: a review of the literature. Respir Med. 2002;96(1):1-6. doi: 10.1053/rmed.2001.1207

12. Ferrans VJ, Yu ZX, Nelson WK, Valencia JC, Tatsuguchi A, Avila NA, et al. Lymphangioleiomyomatosis (LAM): a review of clinical and morphological features. J Nippon Med Sch. 2000;67(5):311-29. doi: 10.1272/jnms.67.311

13. Chu SC, Horiba K, Usuki J, Avila NA, Chen CC, Travis WD, et al. Comprehensive Evaluation of 35 Patients with Lymphangioleiomyomatosis. Chest. 1999;115(4):1041-52. doi: 10.1378/chest.115.4.1041

14. Nakatsu M, Hatabu H, Morikawa K, Uematsu H, Ohno Y, Nishimura K, t al. Large coalescent parenchymal nodules in pulmonary sarcoidosis: "sarcoid galaxy" sign. AJR Am J Roentgenol. 2002;178(6):1389-93. doi: 10.2214/ajr.178.6.1781389

15. Reddy GP, Ahuja JP. Thoracic Sarcoidosis: Imaging Patterns. Semin Roentgenol. 2019;54(1):59-65. doi: 10.1053/j.ro.2018.12.008
16. Aluja-Jaramillo F, Gutiérrez FR, Rossi S, Bhalla S. Nonneoplastic Tracheal Abnormalities on CT. CDR. 2020;43(13):7-8. doi: 10.1097/01.CDR.oooo668644.62958.46

17. Massey H, Darby M, Edey A. Thoracic complications of rheumatoid disease. Clin Radiol. 2013;68(3):293-301. doi: 10.1016/j.crad.2012.07.007

18. Turesson C, Fallon WM, Crowson CS, Gabriel SE, Matteson EL. Extraarticular disease manifestations in rheumatoid arthritis: incidence trends and risk factors over 46 years. Ann Rheum Dis. 2003;62(8):722-7. doi: 10.1136/ard.62.8.722

19. Prete M, Racanelli V, Digiglio L, Vacca A, Dammacco F, Perosa F. Extra-articular manifestations of rheumatoid arthritis: An update. Autoimmun Rev. 2011;11(2):123-31. doi: 10.1016/j.autrev.2011.09.001

20. Copobianco J, Grimberg A, Thompson BM, Antunes VB, Jasinowodolinski D, Meirelles GS. Thoracic manifestations of collagen vascular diseases. Radiographics. 2012;32(1):33-50. doi: 10.1148/rg.321105058

21. Franquet T. High-resolution ct of lung disease related to collagen vascular disease. Radiol Clin N Am. 2001;39(6):1171-87. doi: 10.1016/soo33-8389(05)70337-7

22. Lieberman-Maran L, Orzano IM, Passero MA, Lally EV. Bronchiectasis in Rheumatoid Arthritis: Report of Four Cases and a Review of the Literature Implications for Management with Biologic Response Modifiers. Semin Arthritis Rheum. 2006;35(6):37987. doi: 10.1016/j.semarthrit.2006.02.003

23. Rossi SE, Erasmus JJ, McAdams HP, Sporn TA, Goodman PC. Pulmonary Drug Toxicity: Radiologic and Pathologic Manifestations. RadioGraphics. 2000;20(5):1245-59. doi: 10.1148/radiographics.20.5.gooseo81245

24. Wallis RS, Broder MS, Wong JY, Hanson ME, Beenhouwer DO. Granulomatous Infectious Diseases Associated with Tumor Necrosis Factor Antagonists. Clin Infect Dis. 2004;38(9):1261-5. doi: 10.1086/383317

25. British Thoracic Society Standards of Care Committee. BTS recommendations for assessing risk and for managing Mycobacterium tuberculosis infection and disease in patients due to start antiTNF-alpha treatment. Thorax. 2005;60(10):800-5. doi: 10.1136/thx.2005.046797

26. Dalakas MC. Inflammatory muscle diseases: a critical review on pathogenesis and therapies. Curr Opin Pharmacol. 2010;10(3):346-52. doi: 10.1016/j.coph.2010.03.001 
27. Hallowell R, Ascherman D, Danoff S. Pulmonary Manifestations of Polymyositis/Dermatomyositis. Semin Respir Crit Care Med. 2014;35(2):239-48. doi: 10.1055/s-0034-1371528

28. Owens GR, Follansbee WP. Cardiopulmonary manifestations of systemic sclerosis. Chest. 1987;91(1):118-27. doi: 10.1378/chest.91.1.118

29. Hamaguchi Y. Autoantibody profiles in systemic sclerosis: predictive value for clinical evaluation and prognosis. J Dermatol. 2010;37(1):42-53. doi: 10.1111/j.1346-8138.2009.00762.x

30. Stojan G, Baer AN, Danoff SK. Pulmonary manifestations of Sjögren's syndrome. Curr Allergy Asthma Rep. 2013;13(4):354-60. doi: 10.1007/s11882-013-0357-9

31. Obermoser G, Sontheimer RD, Zelger B. Overview of common, rare and atypical manifestations of cutaneous lupus erythematosus and histopathological correlates. Lupus. 2010;19(9):1050-70. doi: 10.1177/0961203310370048

32. Goh YP, Naidoo P, Ngian GS. Imaging of systemic lupus erythematosus. Part I: CNS, cardiovascular, and thoracic manifestations. Clin Radiol. 2013;68(2):181-91. doi: 10.1016/j.crad.2012.06.110

33. Paran D, Fireman E, Elkayam O. Pulmonary disease in systemic lupus erythematosus and the antiphospholipid syndrome. Autoimmun Rev. 2004;3(1):70-5. doi: 10.1016/S1568-9972(03)ooo9o-9

34. Yeung H, Takeshita J, Mehta N, Kimmel SE, Ogdie A, Margolis DJ, et al. Psoriasis severity and the prevalence of major medical comorbidities: a population-based study. JAMA Dermatol. 2013;149(10):1173-9. doi: 10.1001/jamadermatol.2013.5015

35. Wu CY, Hu HY, Li CP, Chou YJ, Chang YT. Comorbidity profiles of psoriasis in Taiwan: A latent class analysis. PLoS ONE. 2018;13(2):e0192537. doi: 10.1371/journal.pone.0192537

36. Balsi DD, Celik E, Genc S, Celik MM, Inan MU. Impaired Pulmonary Function in Patients with Psoriasis. Dermatology. 2006;232(6):664-7. doi: 10.1159/000456032

37. Londoño A, González C, Castro L, Puig L. Psoriasis y su relación con el síndrome metabólico. Rev Colomb Reumatol. 2013;20(4):228-36.

38. Chiang H, Lin H. Association between psoriasis and chronic obstructive pulmonary disease: a population-based study in Taiwan. J Eur Acad Dermatol Venereol. 2012;26(1):59-65. doi: 10.1111/j.1468-3083.2011.04009.x
39. Ungprasert P, Srivali N, Thongprayoon C. Association between psoriasis and chronic obstructive pulmonary disease: A systematic review and meta-analysis. J Dermatolog Treat. 2016;27(4):31621. doi: 10.3109/09546634.2015.1107180

40. Choi Y, Shannon F, Wu J. Incidence of Pulmonary Arterial Hypertension in Patients with Psoriasis: A Retrospective Cohort Study. Perm J. 2017;21:16-073. doi: 10.7812/TPP/16-073

41. Wanat K, Schaffer A, Richardson V, VanVoorhees A, Rosenbach M. Sarcoidosis and Psoriasis A Case Series and Review of the Literature Exploring Co-Incidence vs Coincidence. JAMA Dermatol. 2013;149(7):848-52. doi: 10.1001/jamadermatol.2013.4256

42. Khalid U, Hilmar GH, Hansen PR. Sarcoidosis in Patients with Psoriasis: A Population- Based Cohort Study. PLoS ONE. 2014;9(10):e109632. doi: 10.1371/journal.pone.0109632

43. Restrepo CS, Martínez S, Lemos JA, Carrillo JA, Lemos DF, Ojeda P, et al. Imaging Manifestations of Kaposi Sarcoma. RadioGraphics. 2006;26(4):116985. doi: 10.1148/rg.264055129

44. Willatt HJ, Moyano NC, Apey RC, Lidid AL. Sarcoma de Kaposi extratorácico: evidencias de una enfermedad multisistémica. Rev Chil Radiol. 2010;16(2):80-5. doi: 10.4067/So717-93082010000200008

45. Wolff SD, Kuhlman JE, Fishman EK. Thoracic Kaposi sarcoma in AIDS: CT findings. J Comput Assist Tomogr. 1993;17(1):60-2. doi: 10.1097/00004728-199301000-00010

46. Murdoch DR, Corey GR, Hoen B, Miró JM, Fowler VG Jr, Bayer AS, et al. Clinical presentation, etiology, and outcome of infective endocarditis in the 21st century: the International Collaboration on Endocarditis-Prospective Cohort Study. Arch Intern Med. 2009;169(5):463-73. doi: 10.1001/archinternmed.2008.603

47. Dodd JD, Souza CA, Müller NL. High-Resolution MDCT of Pulmonary Septic Embolism: Evaluation of the Feeding Vessel Sign. AJR Am J Roentgenol. 2006;187(3):623-9. doi: 10.2214/AJR.05.0681

48. Depasquale R, Kumar N, Lalam RK, Tins BJ, Tyrrell PNM, Singh J, et al. SAPHO: What radiologists should know. Clin Radiol. 2012;67(3):195-206. doi: 10.1016/j.crad.2011.08.014

49. Lew PP, Ngai SS, Hamidi R, Cho JK, Birnbaum RA, Peng DH, et al. Imaging of Disorders Affecting the Bone and Skin. RadioGraphics. 2014;34(1):197-216. doi: 10.1148/rg.341125112 